http://dx.doi.org/10.15198/seeci.2017.43.15-27

Recibido: 17/01/2017 --- Aceptado: 19/03/2017

\title{
INTELIGENCIA EMOCIONAL Y EDUCACIÓN UNIVERSITARIA: UNA APROXIMACIÓN
}

\section{EMOTIONAL INTELLIGENCE AND HIGHER EDUCATION: A CLOSER VIEW}

José Ignacio Niño González¹. Universidad Complutense de Madrid. España. josenino@ucm.es

Enrique García García. Periodista. España.

garcicomunicacion@gmail.com

David Caldevilla Domínguez. Universidad Complutense de Madrid. España. davidcaldevilla@ccinf.ucm.es

\section{RESUMEN}

Se busca establecer la importancia y atención que se presta actualmente a la educación emocional en las instituciones superiores: su valor para la vida y formación de los alumnos y para su éxito personal y profesional, así como el esfuerzo que se ha estado haciendo en las instituciones educativas para potenciar este juego de competencias. Igualmente nos interesa la percepción de las habilidades sociales de estos por parte de los agentes académicos y laborales. Antes de todo ello, determinaremos el origen de la inteligencia emocional como concepto y la evolución de la percepción académica de inteligencia, desde los tiempos en que lo determinaba el dominio de materias académicas, hasta los test de CI y la inteligencia emocional. De esta manera podremos comprobar cuáles han sido los criterios empleados para medir el intelecto humano y compararlos entre sí para poder preguntarnos de manera efectiva por el grado de validez de nuestras propias ideas actuales en la materia. Al tener una idea más clara de los esfuerzos que global e históricamente se han hecho en este campo, tendremos una mejor posición desde la cual juzgar la calidad y cantidad de esfuerzo destinado tanto a la medición de la capacidad intelectual como de la capacidad de interacción.

\section{PALABRAS CLAVE}

Inteligencia emocional - Universidad - Modelos de comunicación - Gestión de emociones - MEIS - Rendimiento académico - Habilidades sociales.

\footnotetext{
1José Ignacio Niño: Profesor Asociado en el Departamento de Comunicación Audiovisual y Publicidad II de la Facultad de Ciencias de la Información, Universidad Complutense de Madrid, impartiendo la asignatura "Investigación y Planificación de Medios".

Correo: josenino@ucm.es
} 


\section{ABSTRACT}

We seek to stablish the importance and attention that is being paid today to emotional education in high educational institutions: it's worth for life and the student's formation, for their personal and professional success. Also the effort that has been made in the educational institutions to empower these set of competences. In the same way we are interested in the perception of social abilities that is reaching the academic and professional social agents. Aside all of this, we'll determine the origin of emotional intelligence as a concept and the evolution of the academic concept of intelligence, from the times in which it was determined by the mastering of academic subjects, to those characterized by IC and emotional intelligence tests. This way, we could be able to see what has been the criteria employed for the measurement of human intellect and compare them, so we can ask ourselves effectively about the degree of our own, today's ideas in the subject. Having a clearer idea about the global and historical evolutions in this field, we'll have a better position from which to judge the quality and quantity of work aimed to the measurement of both de Intellectual Capacity and the interaction abilities.

\section{KEY WORDS}

Emotional Intelligence - University - Communication models - Emotion management - MEIS - Academic Performance - Social skills.

\section{Cómo citar el artículo}

Niño González, J. I., García García, E. y Caldevilla Domínguez, D. (2017). Inteligencia emocional y educación universitaria: una aproximación [Emotional intelligence and higher education: a closer view] Revista de Comunicación de la SEECI, 43, 15-27. http://dx.doi.org/10.15198/seeci.2017.43.15-27

Recuperado de http://www.seeci.net/revista/index.php/seeci/article/view/486

\section{1.- INTRODUCCIÓN}

La reflexión y la comunicación -entendida como comunicación eficiente y generadora de respuestas positivas- son dos actividades intrínsecamente ligadas por la necesidad de dar salida a mensajes que cargan con el lastre de la subjetividad de cada individuo. Ahí entran en juego, de manera combinada y no reglada, la naturaleza y fuerza de los sentimientos implicados, la claridad del razonamiento expuesto y las habilidades comunicativas de emisor y receptor.

Cuando se une el concepto cognitivo al emotivo, autores como Redó (2010) centran sus estudios en combinar la fisiología y el pensamiento:

El neurobiólogo Damasio (2001), cuyos estudios sobre el cerebro confirman que el sistema límbico y la corteza se influyen entre sí, explica cómo la emoción influye sobre la cognición, y especialmente sobre la memoria. Recordamos determinados episodios vivenciales pero olvidamos otros. Al hablar de la memoria es preciso destacar que no solo se relaciona con el 
hecho de retener cierta información sino que la memoria forma parte de casi todos los procesos cognitivos: la percepción, toma de decisiones, aprendizaje, planificación, establecimiento de prioridades, creatividad, etc. Por ello tiene un amplio impacto la influencia de la emoción en la cognición. En esa misma línea LeDoux (1999) ha demostrado que es posible estudiar la emoción del modo en que se ha estudiado la razón; es decir analizando cómo el cerebro procesa estímulos. Cuando se trata de estímulos emocionales se produce una respuesta emocional. Sus investigaciones le han llevado al convencimiento que la emoción llega a controlar el pensamiento. Lo argumenta considerando que la emoción es más fuerte que la razón. Es decir, es posible que la razón llegue a controlar la reflexión, y en cambio es muy difícil que el pensamiento racional controle la emoción. Por ello, ante casos de ansiedad o depresión, la razón puede reflexionar sobre lo que está pasando y puede desear decir basta, pero casi nunca lo consigue, puesto que eliminar el estrés o la ansiedad pasa necesariamente por la gestión emocional. Por su parte, Maturana (2001) desde el punto de vista biológico ha definido a las emociones como las "disposiciones corporales dinámicas que definen los distintos dominios de acción en que nos movemos". De ahí que cuando uno cambia de emoción cambia de dominio de acción. Es decir, si cambian nuestras circunstancias emocionales cambia nuestra forma de razonar. (Redó, 2010, p. 79)

A partir de trabajos como éste, se desprende que la actuación y la comunicación humana no pueden ser separadas de manera estanca en actuaciones movidas de manera exclusiva por la razón o por la emoción, aunque existan acciones motivadas de manera exclusiva por las emociones, con muy poca intervención de la racionalidad. Ambos son componentes en una u otra proporción, de todas nuestras iniciativas (Sánchez Leyva, 2015) (Antón Hurtado, 2015).

Siguiendo de nuevo a Maturana (1993), convenimos que el mismo lenguaje oral está cargado, además, con un fuerte componente emocional propio del lenguaje corporal. Ello lleva a que la comunicación corporal resulte un lenguaje mucho más universal: el lenguaje de las emociones trasciende al racional, llega atravesando barreras culturales -no sin dificultades- y aporta valor añadido a aquellos preparados para entenderlo más allá de sus rudimentos esenciales.

Todo lo anterior se ve refrendado por autores como Gergen (1996) cuando afirma que la comunicación social está fundamentada en la interacción verbal, como medio principal también de transmisión de emociones y sentimientos, tanto positivos como negativos, verdaderos o falsos. El lenguaje tiene, para él, carácter generativo pues es capaz de describir realidades y, por lo mismo, de crearlas. Es decir: resulta posible enunciar los acontecimientos, pero también hacerlos suceder mediante el lenguaje.

El lenguaje tiene, como hemos visto, consecuencias en la realidad, empezando por el ambiente emocional generado en torno a los discursos; y la habilidad para utilizar esta capacidad generadora la aprendemos desde muy temprano: aprendemos a gestionar emociones a través del lenguaje. Éste les da forma y las hace 
comprensibles para el intelecto, permitiéndonos verbalizar y racionalizar hasta cierto punto esa parte de nosotros mismos y de los demás.

Esta capacidad de acción comunicativa a través de la oralidad, es esencialmente inteligencia social de relaciones. Según Bruner (1998) dicha inteligencia tiene la habilidad básica de construir creencias incluso sobre las creencias de otros 0 atribuírselas a conceptos 0 individuos para diferenciarlas de las creadas domésticamente. Ello configuraría como rasgo más característico de la Humanidad el de la habilidad de releer y reinterpretar constantemente nuestra capacidad intelectual y nuestras ideas.

Es desde aquí desde donde emana en su totalidad el proceso de socialización y cooperación del que se desprende a su vez la actividad cognitiva. El enfoque sistémico, Bronfenbrenner (1986), establece las bases para caracterizar las relaciones entre seres humanos como relaciones entre sistemas abiertos (otras personas, pero también familias, grupos de amigos, centros de trabajo y estudios, etc.). Por su parte, el lenguaje escrito se diferencia del oral en que supone una abstracción representativa del pensamiento, y alude a momentos comunicativos diferentes, por lo que carece del contexto de la situación. Nosotros mismos nos vemos obligados a crear el contexto: a crearlo mediante el lenguaje y su influencia en el pensamiento. El recurso a la palabra escrita implica una actitud más independiente, voluntariosa y libre con respecto a ella.

Así, Vigotsky los clasifica como herramientas y signos; los primeros serían instrumentos concretos que facilitan las relaciones externas con la naturaleza, pluma, bolígrafo o procesador de textos-; los signos son instrumentos en el nivel psicológico, de carácter interior, conformados por el sistema escrito en sí, por el código concreto entendido como idioma, por el alfabeto, etc. La escritura es más compleja que la comunicación oral porque no puede darse de manera subconsciente ni irreflexiva.

\section{OBJETIVOS}

Se busca determinar la importancia que se da y la atención que se concede a la educación emocional en las instituciones de educación superior: su valor para el desarrollo vital y formativo de los estudiantes, así como para el desarrollo de sus expectativas de crecimiento en el ámbito laboral y humano. Todo ello incardinado dentro del marco referencial sobre el esfuerzo que se ha efectuado en las instituciones educativas para crear un sencillo pero efectivo juego de competencias entre el alumnado. Igualmente profundizamos sobre cuál es la percepción de las habilidades sociales de éstos en las esferas empresarial y universitaria.

In concreto, en el presente artículo nos centraremos en los modelos de comunicación interpersonal que existen en la enseñanza de hoy, de manera que podamos analizar la huella de la inteligencia emocional para ponderar su importancia en la docencia. 


\section{METODOLOGÍA}

El presente artículo parte de una necesidad de conocimiento inicialmente sustentada sobre un exhaustivo análisis de fuentes, o hermenéutica, para determinar el statu $q u o$ actual de la formación en inteligencia emocional. Sobre esta base, aplicamos un proceso inductivo-deductivo, basado tanto en los citados estudios de fuentes como de casos actuales tomados como base de las nuevas teorías.

Los actuales estudios focalizan el análisis de la inteligencia emocional (objeto de estudio) como medio (vehículo) para transmitir otros saberes humanísticos propios de una publicación científica del área de Humanidades.

Nuestra labor consistirá en discernir cuáles de estos conocimientos es el adecuado para su aplicación en el campo de la enseñanza superior. Con la nueva implantación del EEES (o Plan Bolonia) los contenidos curriculares han mudado hacia la tecnología en tanto en cuanto herramienta y objeto de estudio (las famosas TIC), pero dejando a un lado las competencias emocionales propias del aprendizaje.

A fin de no obviar ninguno de los aspectos conformantes del proceso comunicativo, centraremos nuestro método en un análisis de contenido de los principales estudios desarrollados en los últimos años y que se configuran ya como modelos clásicos de estudio del fenómeno comunicativo interpersonal y de la inteligencia emocional.

La metodología empleada se basa en analizar dónde aciertan y donde fallan estos aspectos a la hora de implantar un nuevos sistema de aprendizaje que se pretende colaborativo (es decir, que exige la aplicación de la inteligencia emocional) pero que puede adolecer de algunos elementos clave para su correcto desarrollo, lo cual conforma nuestra hipótesis de trabajo.

\section{DISCUSIÓN}

\subsection{Modelos de comunicación en la enseñanza}

La influencia mutua entre comunicación y relación interpersonal plantea la necesidad de ahondar en el conocimiento del estilo de comunicación de cada persona. Subconscientemente todos tenemos en nuestro interior diversos modelos comunicativos. El contexto y la inteligencia emocional determinan cuál de ellos tendrá preeminencia en un momento comunicativo dado. La individualidad de cada uno lleva a recurrir a un modelo u otro, siendo estos a grandes rasgos, siguiendo el modelo establecido por Redó (2010).

- Modelo dominante

- Modelo sumiso

- Modelo cooperador

Dichos modelos no afloran "en estado puro": las respuestas son una mezcla de los tres estilos, en las que uno de ellos es el preponderante. La composición exacta de la respuesta se estructura de manera orgánica a la situación concreta que se esté 
abordando, las personas implicadas, la relación con y entre las mismas, etc. Aunque pudiera parecer lo contrario, el estilo "cooperador" es el habitualmente menos preponderante; si bien buena parte de los licenciados universitarios dicen ser competentes en materia de trabajo en equipo -de lo que se desprendería lo contrario- esta capacidad no es percibida por los empleadores dentro del mismo grupo de referencia (Barraycoa y Lasaga, 2009 y 2010). Otro indicador negativo en este sentido, lo daría el escaso interés que demuestra la Administración por la enseñanza de materias de Humanidades, como la filosofía o la ética, que podrían resultar importantes en este sentido (Del Campo Lozano, 2012). La complejidad inherente a las comunicaciones interpersonales hace que, de hecho, la satisfacción mutua de las necesidades de dos partes sea raramente sencilla. Ello es, en no poca medida, debido a que no existe una preparación colectiva para ello: desde la infancia, y cada vez más, recibimos mensajes educacionales de carácter competitivo. La inmediatez predominante en las comunicaciones nos impulsa, además, a buscar soluciones de comunicación inmediatas, poco meditadas e incluso estandarizadas, por poco acertadas que éstas puedan llegar a ser desde el punto de vista racional, siempre según Lozano.

Ello lleva a plantear el potencial de la comunicación como materia educativa en la formación no ya académica sino humana. En este sentido, es crucial potenciar el poco explotado modelo cooperativo de comunicación ya desde los primeros pasos de la educación académica, que es por ende social, y la aprehensión del lenguaje, de manera que cada individuo tuviese herramientas más desarrolladas para dar lo mejor de sí mismo y recibirlo de los demás en sus relaciones sociales. Ello requeriría un esfuerzo consciente en el ámbito escolar en particular y en el proceso educativo en general. De hecho, los alumnos entienden desde muy temprana edad que están rodeados de iguales, y que de la misma manera que ellos, aquéllos tienen sentimientos, creencias, sufrimientos y pareceres propios. Desde este punto de partida, es factible ejemplificar ante los estudiantes, por parte de sus profesores, un modelo cooperativo de comunicación inteligente.

\subsection{Inteligencia emocional}

Trabajamos en torno a un concepto que ha re-definido la idea misma de la inteligencia: desde su concepción primitiva de la escuela clásica, en la que la inteligencia venía definida por el dominio de una serie de materias (matemáticas, griego, latín, física, etc.) hasta la más actual, basada en una mezcla de rendimiento académico y puntuación de Cociente Intelectual. Esta idea ha quedado obsoleta para algunos autores, que son de la opinión de que la inteligencia académica tradicional no es suficiente para alcanzar el éxito profesional, puesto que los profesionales más prestigiosos lo son a causa de su capacidad de reconocer y administrar las emociones propias y ajenas con mayor eficacia. Por lo mismo, consideran que la inteligencia entendida como cociente intelectual bruto no se traduce necesariamente en éxito vital, ni da señales del equilibrio mental o emocional de la persona. (Fernández-Berrocal y Extremera, 2003)

Se plantea en primera instancia, como medir este tipo de inteligencia de manera fiable. Existen tres grupos de métodos principales, según Suberviola-Ovejas (2012): 
- Cuestionarios, escalas y auto-informes:

Este método muy habitualmente utilizado. Entre las más conocidas cabe mencionar el Trait Meta-Mood Scale (TMMS) de Salovey, Mayer, Goldman, Turvey y Palfai (1995) y el TMMS-24 (versión resumida en español, Fernández-Berrocal, Extremera y Ramos, 1999). En todas ellas se persigue una aproximación individual sobre los aspectos reflexivos del hecho emocional.

- Observadores externos:

Parte del presupuesto de que si la IE implica la capacidad para manejar y comprender nuestras propias emociones y las de las personas que nos rodean, ¿por qué no preguntar a las personas próximas a nosotros sobre cómo manejamos nuestras emociones en público y la forma en que afrontamos los problemas y sucesos que nos suceden? (Extremera y Fernández-Berrocal, 2004).

- Tareas emocionales o de habilidad:

Pretende eliminar la parcialidad a la que son susceptibles otros métodos. También esquivar las respuestas adulteradas en función de la necesidad de dar una imagen positiva en detrimento de una imagen precisa.

En este sentido, Alarcón García y Guirao Mirón (2014) concluyen:

En cuanto a las competencias del EEES, no es fácil dar una definición única de competencia, debido al carácter multidimensional del concepto que agrupa elementos actitudinales, comportamentales, conceptuales y sociales (Colás 2005). No obstante hay que decir que la mayoría de los autores convienen en que la competencia no es solo un saber hacer, sino que convergen en ella el "saber" con el "saber hacer" pues no es algo meramente instrumental, hay que comprender e interiorizar por que y para que (Delors, 1996; Colás, 2005; ANECA, 2005 Zabala y Arrnau, 2008).Las competencias posibilitan, fundamentalmente, que la persona que las desarrolla sea capaz de ejercer una actividad o profesión. En este sentido, el modelo de competencia universitaria corre el riesgo de centrar el aprendizaje universitario en torno a productos formativos, a competencias profesionales y de cara al mercado laboral, en detrimento del desarrollo de otras competencias más sociales, emocionales, cívicas o morales. Creemos que el concepto de competencia debe ser indisociable del desarrollo integral de la persona en la línea de la teoría del enfoque de las capacidades, en la que las emociones tienen un lugar preciso.

Un individuo dotado de competencias emocionales ampliamente desarrolladas es lo que llamaríamos empático: es consciente de las emociones propias y ajenas a través de la comunicación: las comprende y sabe cómo debe actuar ante ellas, obteniendo de ese modo la habilidad para administrarlas de la mejor manera posible.

Numerosos son los estudios centrado en la relación comprobada entre la Inteligencia Emocional y el desenvolvimiento social: una mayor capacidad para reconocer expresiones faciales y no verbales emocionales, una mayor percepción de aceptación social y más satisfacción con el establecimiento de relaciones dentro del entorno (Ciarrochi; Chan \& Caputi, 2000). Una nota alta en Inteligencia Emocional obtenida 
con MEIS se vincula a un nivel superior de empatía (Mayer; Caruso y Salovey, 1999). $Y$ finalmente, Extremera y Berrocal (2004) proponen que un mejor juego de aptitudes emocionales se traduce en un mayor porcentaje de interacciones sociales exitosas, resultando en niveles de bienestar personal más acusados en el sentido positivo.

\subsection{Importancia en el rendimiento académico}

Siguiendo a Palomera, Fernández-Berrocal y Brackett (2008):

Las emociones y las habilidades relacionadas con su manejo, afectan a los procesos de aprendizaje, a la salud mental y física, a la calidad de las relaciones sociales y al rendimiento académico y laboral (Brackett y Caruso, 2007). La docencia es considerada una de las profesiones más estresantes, sobre todo porque implica un trabajo diario basado en interacciones sociales en las que el docente debe hacer un gran esfuerzo para regular no solo sus propias emociones sino también las de los estudiantes, padres, compañeros, etc. (Brotheridge y Grandey, 2002). Los profesores, desafortunadamente, experimentan con más frecuencia emociones negativas que positivas (Emmer, 1994). Las emociones negativas, como por ejemplo la ansiedad interfieren en nuestra capacidad cognitiva para el procesamiento de la información (Eysenck y Calvo, 1992) mientras que las positivas, aumentan nuestra capacidad creativa para generar nuevas ideas y por tanto nuestra capacidad de afrontamiento ante las dificultades (Frederickson, 2001). Las emociones positivas de los profesores pueden aumentar el bienestar docente y también el ajuste de sus alumnos (Birch y Ladd, 1996). Este efecto positico además puede formar una espiral que a su vez facilite un clima de clase más favorecedor para el aprendizaje (Sutton y Whealey, 2003). Es por ello que la capacidad de identificar, comprender y regular las emociones, tanto positivas como negativas, se hace imprescindible en esta profesión, para poder utilizar y generar las emociones a nuestro favor.

La importancia de la Inteligencia Emocional en el Ámbito educativo, y sobre todo la que se trasluce como un factor fuerte en el éxito académico y en la integración de los estudiantes en la sociedad externa a la Universidad, es lo que más se valora actualmente en el estudio de las materias curriculares (Extremera y Berrocal, 2004). Aun estando hoy clara la relevancia y preferencia de la comprensión sobre la memorización en el aprendizaje, es preciso ir más allá y ampliar las competencias emocionales del alumnado, debido al impacto que éstas tienen tanto a nivel educativo como social. La mayoría de los estudios señalan la relación entre Inteligencia Emocional, Competencia social y éxito académico. (Fernández-Berrocal \& Extremera, 2006). Sin embargo, y como dato, las conclusiones aportadas por Ortiz Sobrino \& Rodríguez Barba (2011) indican que la oferta de formación en Inteligencia Emocional no forma parte de los referentes del alumnado a la hora de elegir carrera o centro de estudios superiores. Las habilidades emocionales podrían contribuir a la adaptación académica y social de varias formas: por ejemplo, como promotoras del proceso mental, puesto que el trabajo cognitivo supone emplear y administrar las 
emociones en favor de la concentración, el control de la presión y, en general, de un incremento en la motivación del estudiante para llevar a cabo sus estudios y todas las actividades que se relacionan con ellos (Maestre, 2006). La Inteligencia Emocional también podría influir sobre el rendimiento académico, haciendo destacar al alumnado emocionalmente competente en ciertas materias, como literatura 0 asignaturas plásticas (Petrides; Frederickson \& Furnhan, 2004). Incluso la gestión de equipos se basa en gran parte en el dominio de este tipo de inteligencia (Fainstein, 1997).

En definitiva, hay evidencias suficientes de la importancia del estado y la gestión emocional en el rendimiento del alumno, así como del papel directo de la Inteligencia emocional en el rendimiento y el bienestar de este en el entorno académico y social. (Extremera \& Fernández-Berrocal, 2003) (Jiménez, 2009).

\section{CONCLUSIONES}

Cualquier esfuerzo destinado a la promoción de la inteligencia emocional en las aulas universitarias es, en última instancia, inútil si no es percibida como fundamental por parte de las empresas y de los agentes que demandan esta serie de capacidades como parte de sus requisitos curriculares en la contratación de nuevos licenciados, allende sus capacidades y habilidades ad hoc.

Dichos esfuerzos chocan también con una cultura empresarial cambiante y la contracultura de la competición sobre la cooperación, que promueve modelos de comunicación interpersonal distintos al cooperador, ya de por si infrautilizado. Los estudios hablan inequívocamente de la ventaja que un adecuado juego de habilidades empáticas otorga a un trabajador $\mathrm{O}$ estudiante sobre otro. $\mathrm{Y}$, sin embargo, los modelos educativos actuales no contemplan estas expectativas socializadoras. Sin duda, estamos hablando de capacidades difíciles de adquirir de manera no planificada, y todo enfoque que pretenda enseñarlas exclusivamente en las instituciones superiores -olvidando las primarias- no resulta realista. Una educación emocional completa pasa por un cambio razonado y razonable del sistema educativo desde la base misma del sistema, a fin de implantar un modelo cooperativo de relación, de forma que fuese posible contar con un mayor número de docentes capacitados para aplicarlo adecuadamente. Este nuevo modelo se aplicaría tanto a largo como a corto plazo, pues implica un esfuerzo adicional por parte del profesorado encargado de manejar este modelo -dada su mayor complejidad frente al sistema actual- lo que forzosamente debería conllevar, desde un punto de vista social, a una revisión de valores sobre un nuevo modelo social.

\section{BIBLIOGRAFÍA}

Alarcón García, G. y Guirao Mirón, C. (2014). ¿Donde están las competencias del EEES en el desarrollo de las emociones, de la inteligencia emocional?, en $\mathrm{J}$. Rodríguez Torres, (Coord.) Experiencias en la adaptación al EEES. Madrid: McGraw Hill Education. Recuperado de https://goo.gl/XvpQLw

Anton Hurtado, F. (2015). Antropología del miedo. Methaodos. Revista de ciencias sociales, 3 (2), 262-275. doi: http://dx.doi.org/10.17502/m.rcs.v3i2.90 
Niño, J. I. García, E. Caldevilla, D. Inteligencia emocional y educación universitaria: una aproximación

Arís Redó, N. (2010). La educación emocional y la comunicación escolar. Vivat Academia, Revista de comunicación, 113, 78-87. doi: https://doi.org/10.15178/va.2010.113.79-87

Barraycoa Martínez, J. \& Lasaga Millet, O. (2010). La competencia de trabajo en equipo: más allá del corta y pega. Vivat Academia, Revista de comunicación, 111, 65-69. doi:https://doi.org/10.15178/va.2010.111.65-69

Barraycoa, J. \& Lasaga, O. (2009). Competencias e inserción laboral: un análisis de la empleabilidad en los recién licenciados en Ade y Economía. Madrid: CEU Ediciones.

Birch, S.H. y Ladd, G.W. (1996). Interpersonal relationships in the school environment and children's early school adjustment: The role of teachers and peers. En J. Juvenon y K. Wentzel (Eds.), Social motivation: Understanding children's school adjustment (pp.199-225). New York: Cambridge University Press.

Brackett, M.A. y Caruso, D.R. (2007). Emotionally literacy for educators. Cary, NC: SELmedia.

Bronfenbrenner, U. (1986). Ecología del desarrollo humano. Barcelona: Paidós.

Brotheridge, C.M. y Grandey, A.A. (2002). Emotional intelligence and burnout: Comparing two perspectives of 'people work'. Journal of Vocational Behavior, 60, 17-39.

Bruner, J. (1998). Realidad mental y mundos posibles. Barcelona: Gedisa.

Ciarrochi, J. V.; Chan, A. \& Caputi, P. (2000). A critical evaluation of the emotional intelligence construct. Personality and Individual differences, 28(3), 561-570. doi: https://doi.org/10.1016/S0191-8869(99)00119-1

Del Campo Lozano, J. D. (2012). Objetivos pedagógicos básicos en la enseñanza de la ética de la comunicación. Vivat Academia, Revista de Comunicación, 121, 116. doi:https://doi.org/10.15178/va.2012.121.1-16.

Emmer, E.T. (1994). Toward an understanding of the primary of classroom management and discipline. Teaching Education, 6, 65-69.

Extremera, N. \& Fernández-Berrocal, P. (2003). La inteligencia emocional en el contexto educativo: Hallazgos científicos de sus efectos en el aula. Revista de Educación, 332, 97-116. doi:https://doi.org/10.4438/1988-592X-0034-8082-RE 
Extremera, N. \& Fernández-Berrocal, P. (2004). ¿Cómo se mide la inteligencia emocional?, en J. L. Zaccagnini Sancho. Que es Inteligencia emocional. La relación entre pensamientos y sentimientos en la vida cotidiana. Madrid: Biblioteca Nueva.

Extremera, N. \& Fernández-Berrocal, P. (2004). Inteligencia emocional, calidad de las relaciones interpersonales y empatía en estudiantes universitarios, Revista Clínica y Salud, 15(2), 117-137. Recuperado de http://emotional.intelligence.uma.es/documentos/PDF6empatia_en_estudiantes_ universitarios.pdf

Eysenck, M.W. y Calvo, M.G. (1992). Anxiety and performance: the processing efficiency theory. Cognitive Emotion, 6, 409-434.

Fainstein, H. (1997). La Gestión de Equipos Eficaces: Organizaciones del Siglo XXI. Buenos Aires: Ediciones Macchi.

Fernández-Berrocal, P. \& Extremera, N. (2006). Emotional Intelligence: A theoretical and empirical review of its first 15 years of history. Psicothema, 18, 7-18. Recuperado de http://www.psicothema.com/pdf/3270.pdf

Frederickson, B.L. (2001). The role of positive emotions in positive psychology: the broadening-and-build theory of positive emotions. American Psychology, 56, 218226.

Gergen, K. (1996). Realidades y Relaciones. Madrid: Paidós.

Jiménez, I. (2009). Una aproximación psicosocial a la relación entre inteligencia emocional y rendimiento académico. Jaén: Universidad de Jaén.

Ledoux, J. (1999). El cerebro emocional. Barcelona: Planeta.

Maestre, J. M.; Güil, R.; Lopes, P.; Salovey, P. \& Gil-Olarte, P. (2006). Emotional Intelligence and Social and academic adaptation to school. Psicothema, 18, 112117. Recuperado de: www.psicothema.com/psicothema.asp?id=3285

Maturana, H. (1993). Amor y Juego, Fundamentos Olvidados de lo Humano. Santiago de Chile: Instituto de Terapia Cognitiva.

Mayer, J.; Caruso, D. R \& Salovey, P. (1999). Emotional intelligence meets traditional standards for an intelligence. Intelligence 27, 267-298. Recuperado de: https://goo.gl/bZUdx2

Ortiz Sobrino, M. Á. \& Rodríguez Barba, D. (2011). El perfil de entrada de los alumnos de grado en las facultades de comunicación de las universidades de Madrid: el caso de la summer media school de la fundación fides/ufv. Vivat 
Niño, J. I. García, E. Caldevilla, D. Inteligencia emocional y educación universitaria: una aproximación

Academia, Revista de Comunicación, 114, 243-276. doi: http://dx.doi.org/10.15178/va.2011.114.243-276

Palomera, R.; Fernández-Berrocal, P.; Brackett A., M. (2008) La inteligencia emocional como una competencia básica en la formación inicial de los docentes: algunas evidencias. Education \& Psycology, 15. Recuperado de http://www.investigacionpsicopedagogica.org/revista/articulos/15/espannol/Art 15 276.pdf

Petrides, K. V.; Frederickson, N. \& Furnhan, A. (2004). The role of trait emotional intelligence in adademic performance and deviant behavior at school. Personality and individual differences, 36(2), 277-293. doi: http://doi.org/10.1016/S0191-8869(03)00084-9

Sánchez Leyva, M. J. (2015). Del "Yo es otro" al "Yo soy yo mismo": emociones y dominación social. Methaodos. Revista de ciencias sociales, 3 (2), 253-261. doi: http://dx.doi.org/10.17502/m.rcs.v3i2.89

Suberviola-Ovejas, I. (2012). Competencia emocional y rendimiento académico en el alumnado universitario. Vivat Academia, Revista de comunicación, 14, 1-17. doi: https://doi.org/10.15178/va.2011.117E.1-17

Sutton, R.E. y Wheatley, K.F. (2003). Teacher's Emotions and Teaching: A review of the literature and directions for future research. Educational Psychology Review, 15(4), 327-358

Vigotsky, L. (1995). Pensamiento y Lenguaje. Nueva edición a cargo de Alex Kozulin. Barcelona: Paidós.

\section{AUTORES}

\section{José Ignacio Niño González}

Profesor Asociado en el departamento de Comunicación Audiovisual y Publicidad II de la Facultad de Ciencias de la Información, Universidad Complutense de Madrid, impartiendo la asignatura "Investigación y Planificación de Medios". Magíster en Gestión Publicitaria impartiendo la asignatura "El advergaming como nuevo medio publicitario" en la Facultad de Ciencias de la Información de la Universidad Complutense de Madrid. Desde 2010, dirige "Global Digital Marketing". Sus principales trabajos de investigación son los proyectos de Innovación y Mejora de la Calidad Docente: "El aula virtual como escenario de mediación para la interculturalidad y los derechos humanos" en la Universidad Complutense de Madrid. http://orcid.org/0000-0003-2775-7241

\section{Enrique García García}

Licenciado en Periodismo, Humanidades y CC. de la Información por la Universidad San Pablo CEU. Máster CES en Periodismo Audiovisual. Director del Magazine "Cultura Pop", ha cubierto todo tipo de información en su paso por la prensa y la 
radio: en el diario "el economista", deportes COPE y sociedad para Radio Intercontinental. Ha participado en investigaciones como "Antonio Gramsci y las raíces lejanas del Eurocomunismo: el precedente olvidado" y "Análisis de la comunicación en las instituciones museísticas madrileñas"

http://orcid.org/0000-0002-1872-5013

\section{David Caldevilla Domínguez}

Licenciado y Doctor en CC.II., (Comunicación Audiovisual por la U. Complutense). Diplomado en Magisterio (U. de Zaragoza). Acreditado a titular (ANECA). Docente en la U. Complutense, U. Europea de Madrid, IED, ESERP e IPAM (Oporto -Portugal-). Ponente y conferenciante y profesor en varios títulos propios (Telemadrid, Walter \& Thompson, McCann...). Secretario General de la SEECI (Sociedad Española de Estudios de Comunicación Iberoamericana) y del "Fórum Internacional de la Comunicación y Relaciones Públicas" (Fórum XXI). Investigador Principal (IP) del Grupo Complutense de Investigación 'Concilium, grupo de comunicación'. Autor de más de 50 artículos científicos, de más de 70 ponencias en Congresos y de 6 libros. Miembro de comités científicos en congresos y revistas científicas internacionales. Director de los Congresos CUICIID. Tertuliano radiofónico. http://orcid.org/0000-0002-9850-1350 